Toutes les personnes impliquées dans la formation postgraduée des spécialistes s'accordent à dire qu'il est important d'évaluer régulièrement le niveau de compétences pour détecter et combler suffisamment tôt les lacunes. Partout dans le monde, l'évaluation en milieu de travail (EMiT) s'est avérée être un instrument approprié pour intégrer une telle évaluation dans les processus hospitaliers sans investissements considérables. La Société suisse de psychiatrie et de psychothérapie (SSPP) a été la première société de discipline à ajouter l'EMiT à son logbook et à l'introduire dans tout le pays. Les documents nécessaires ont été élaborés en collaboration avec l'Institut d'enseignement médical (IML) de l'Université de Berne, qui a également réalisé les ateliers de formation pour les responsables de la formation postgraduée. L'Institut suisse pour la formation médicale postgraduée et continue (ISFM) soutient financièrement l'introduction des EMiT dans le but d'inciter d'autres sociétés de discipline à suivre dès que possible l'exemple des psychiatres.

\title{
Introduction de l'évaluation en milieu de travail (EMiT) par les sociétés de discipline
}

\author{
Stephanie Montagnea, \\ Julius Kurmann ${ }^{b}$, \\ Patrick Jucker-Kuppera, \\ Christine Beyelera, \\ Werner Bauerc \\ a Unité des examens et de \\ l'évaluation (AAE), Institut \\ d'enseignement médical \\ (IML), Université de Berne \\ b Commission permanente \\ pour les formations \\ postgraduée et continue (CPF) \\ de la Société suisse de \\ psychiatrie et psychothérapie \\ (SSPP) \\ c Institut suisse pour la \\ formation médicale \\ postgraduée et continue \\ (ISFM), Berne
}

Liens d'intérêts:

L'Institut suisse pour la formation médicale postgraduée et continue (ISFM) soutient financièrement l'Unité des examens et de l'évaluation (AAE) de l'Institut d'enseignement médical (IML) de l'Université de Berne pour le développement, l'implémentation et l'évaluation de l'EMiT dans la formation postgraduée des médecins.

Correspondance: Dr S. Montagne Unité des examens et de l'évaluation (AAE) Institut d'enseignement médical Université de Berne Konsumstrasse 13 CH-3010 Berne

aba[at]iml.unibe.ch

\section{Contexte et objectifs}

La formation postgraduée en vue d'un titre de spécialiste prépare à l'exercice autonome de la profession. Au fil de leur formation postgraduée, les médecins-assistants élargissent leurs compétences médicales et prennent de plus en plus de responsabilité grâce à leurs activités cliniques et à l'échange permanent avec les formateurs. Dans ce contexte, l'application pratique des connaissances acquises est tout aussi décisive qu'un feed-back régulier par des médecins hospitaliers expérimentés [1]. Cependant, l'enquête menée en 2008 auprès des médecins-assistants sur l'évaluation des établissements de formation postgraduée a montré, toutes disciplines confondues, qu'il fallait encore agir dans ce domaine notamment au niveau des établissements de formation d'une certaine envergure [2]. Etablie dans beaucoup de pays, l'évaluation en milieu de travail (EMiT) est une méthode appropriée à un encouragement ciblé et à une appréciation régulière des compétences médicales. Alors que l'EMiT renseigne les médecins en formation postgraduée sur le niveau des compétences qu'ils ont acquises et les aide à travailler concrètement à les améliorer, elle donne aux formateurs une image plus précise des progrès accomplis par les médecins-assistants dans leur formation. L'EMiT se décline en deux instruments: le Mini Clin-ical Evaluation Exercise (Mini-CEX) [3] pour évaluer principalement les techniques d'examen et les aptitudes communicatives (par ex. anamnèse, examen clinique ou information aux patients), et la Direct Observation of Procedural Skills (DOPS) qui met l'accent sur les aptitudes manuelles [4], tous deux étant fondés sur les piliers «observer» - «documenter» «donner un feed-back». En plaçant un médecin-assistant sous l'observation directe d'un formateur lors d'une rencontre quotidienne avec un patient, l'EMiT vient compléter les instruments traditionnels de la formation postgraduée, comme l'enseignement au chevet du patient ou la supervision psychiatrique. Le formateur discute ensuite avec le médecin-assistant des points forts et des points faibles qu'il a observés et lui indique comment aborder concrètement les lacunes. Cette forme de feed-back structuré, donné pratiquement en temps réel, se laisse facilement intégrer dans les processus de travail quotidiens - ce qui est un facteur important comme l'a montré une enquête réalisée en 2011 auprès des candidats au titre de spécialiste en psychiatrie et psychothérapie [5]. En sollicitant davantage d'échanges entre les formateurs et les médecins-assistants, l'EMiT rend la formation médicale postgraduée plus efficace et en améliore la qualité. Elle renforce également la culture du feed-back, de la communication et de l'erreur dans les hôpitaux [6].

Suite aux expériences acquises lors de la phase pilote lancée depuis quelques années dans plusieurs disciplines [7, 8], l'Institut suisse pour la formation médicale postgraduée et continue (ISFM) a décidé d'appliquer l'EMiT à tout le pays en visant une introduction dans les différentes disciplines au cours des trois prochaines années. L'ISFM en a informé les présidents des sociétés concernées et il a également demandé à l'Institut d'enseignement médical (IML) de l'Université de Berne de les soutenir dans cette démarche.

Le présent compte rendu décrit les procédures appliquées lors de l'introduction de l'EMiT dans les cursus de la Société suisse de psychiatrie et psychothérapie (SSPP). Son objectif est de fournir un exemple concret aux autres sociétés de discipline pour leur indiquer les processus à suivre et leur permettre de profiter des expériences de la SSPP lors de l'introduction de l'EMiT dans leur spécialisation. Notons toutefois que seuls les Mini-CEX sont utilisés en psychiatrie et psychothérapie.

\section{Introduction des Mini-CEX en psychiatrie et psychothérapie}

Le principe des Mini-Clinical Evaluation Exercises (Mini-CEX) et les expériences pratiques dans ce do- 
maine, présentés lors de la Conférence des médecins-chefs en 2010, ont été très bien accueillis, notamment en raison du souhait déterminant de vouloir pérenniser la qualité élevée de la formation postgraduée mais aussi de sensibiliser les formateurs à leur rôle prépondérant dans la formation de la nouvelle génération de spécialistes. La SSPP a donc mandaté un groupe de travail, dirigé par son responsable de la formation postgraduée, le Dr J. Kurmann, pour coordonner l'introduction des Mini-CEX dans les établissements de formation. A ce moment-là, les Mini-CEX apparaissaient déjà dans le logbook mais ils ne faisaient pas encore partie intégrante du programme de formation. La SSPP s'était fixé l'objectif de préparer les établissements de formation de manière optimale à la révision du programme de formation prévue pour 2012

\section{«L'observation directe du médecin assistant par le formateur est à la base de l'EMiT.»}

Composé de cinq représentants des institutions pilotes réparties dans les différentes régions linguistiques et d'une experte de l'IML, le groupe de travail s'est réuni sous la houlette du Dr J. Kurmann et a planifié en deux demi-journées la procédure d'introduction des Mini-CEX. Les membres du groupe de travail se sont également appuyés sur leurs expériences pour valider les documents relatifs aux Mini-CEX. Les grilles d'évaluation, les critères et le matériel d'enseignement mis à disposition lors de la phase pilote ont été retravaillés et standardisés en vue de l'introduction prévue à l'ensemble du pays (voir: http:// emit.iml.unibe.ch/psy/.

Le concept relatif à l'introduction nationale des Mini-CEX prévoit des cours appelés teach the teacher. Proposés dans les différentes régions linguistiques, ils se fondent sur le principe suivant: un ou deux délégués des médecins-cadres d'un établissement de formation postgraduée offrant au moins dix postes de médecins-assistants se rendent à des ateliers interactifs dédiés à leur discipline. Ensuite, ils sont chargés de la formation interne des collaborateurs au sein de leur institution d'origine dans laquelle ils jouent pour ainsi dire un rôle démultiplicateur de ce qu'ils ont appris. La participation à ce cours est sanctionnée par 3 crédits de formation continue et par un certificat de la SSPP.

Le rapport final à l'intention de la SSPP présente les mesures prévues et les recommandations concernant l'introduction des Mini-CEX en psychiatrie et psychothérapie.

Ce processus préparatoire ainsi que les cours teach the teacher ont bénéficié du soutien financier de l'ISFM.

\section{Cours régionaux «teach the teacher»}

Deux cours teach the teacher en allemand ainsi qu'un en français et un en italien, destinés en priorité, comme mentionné plus haut, aux médecins-cadres d'établissements de formation postgraduée offrant au moins dix postes de formation, ont été proposés entre août 2011 et février 2012. Lors d'un atelier de trois heures, les formateurs ont été familiarisés aux principes des Mini-CEX et à leur réalisation. Grâce à des exemples vidéo, ils ont pu s'exercer directement et mettre en pratique notamment la manière de donner un feed-back. Ces ateliers ont été réalisés par des collaborateurs de l'IML en collaboration avec les membres du groupe de travail, qui ont fait partager leur expérience du terrain et répondu aux questions sur les Mini-CEX en lien avec leur discipline. Les exercices pratiques et l'échange entre pairs ont été les éléments importants de ces cours, ce que les participants n'ont pas manqué de reconnaître et de souligner. Ils ont estimé que les cours ont été extrêmement positifs quelle que soit la région linguistique (évaluation générale, note de 1 à 6 , moyenne $=5,2 \pm 0,58$ écart type). En revanche, certains participants ont considéré qu'il était très difficile de transmettre aux collaborateurs de leurs institutions ce qu'ils avaient appris sur les Mini-CEX lors de l'atelier.

Parmi les 47 établissements de formation ayant au moins dix postes de médecins-assistants, 33 ont profité de cette offre, alors que six autres centres avaient déjà été familiarisés avec les Mini-CEX dans le cadre du projet pilote. Cela correspond à une participation de $83 \%$ des établissements de formation de taille moyenne à grande. Lorsque le nombre de places le permettait, les représentants d'établissements de formation plus petits ont également participé à l'atelier. C'est ainsi que 69 (=51\%) établissements sur un total de 136 ont été représentés par une ou deux personnes lors des quatre cours organisés dans les trois régions linguistiques. Précisons qu'en psychiatrie et psychothérapie, une institution regroupe souvent plusieurs établissements de formation (par ex. pour la psychiatrie hospitalière et la psychiatrie ambulatoire ou pour la psychiatrie de la personne âgée). On peut donc en déduire que les médecins-cadres délégués représentaient plus d'un établissement et qu'ils ont ensuite présenté les Mini-CEX à tous les collaborateurs de leur institution. Cependant, cette supposition reste difficile à quantifier car un vaste réseau d'établissements de formation existe en psychiatrie et psychothérapie et ces établissements - bien qu'implantés sur différents sites - appartiennent à une seule et même institution.

\section{Introduction dans les établissements de formation postgraduée en psychiatrie}

Après avoir suivi leur cours régional, les médecinscadres ont donc organisé un cours d'introduction aux Mini-CEX à l'intention des collaborateurs de leur institution. Pour cela, ils ont bénéficié de l'aide 
d'un site internet mis en place à cet effet (http:// emit.iml.unibe.ch/psy/) à partir duquel ils pouvaient télécharger la documentation et les instructions mises à leur disposition par l'IML.

Une étude en ligne a été réalisée auprès de tous les médecins-cadres qui ont participé aux cours en

Tableau 1

Caractéristiques de l'échantillon interrogé.

\begin{tabular}{|c|c|c|}
\hline \multirow{2}{*}{$\begin{array}{l}\text { Caractéristique } \\
\text { Sexe }\end{array}$} & \multicolumn{2}{|c|}{ Nombre et taux sur un total de 57 personnes interrogées } \\
\hline & Femmes & $22(39 \%)$ \\
\hline & Hommes & $35(61 \%)$ \\
\hline \multirow[t]{2}{*}{ Langue } & Allemand & $37(65 \%)$ \\
\hline & Français & $20(35 \%)$ \\
\hline \multirow[t]{5}{*}{ Fonction } & Médecin-chef & $7(12 \%)$ \\
\hline & Médecin adjoint & $19(33 \%)$ \\
\hline & Chef de clinique & $23(40 \%)$ \\
\hline & Autre fonction (par ex. médecin hospitalier) & $2(4 \%)$ \\
\hline & Pas de réponse & $6(10 \%)$ \\
\hline \multirow{3}{*}{$\begin{array}{l}\text { Rôle dans } \\
\text { la formation } \\
\text { postgraduée }\end{array}$} & $\begin{array}{l}\text { Fonction explicite de responsable de la formation } \\
\text { postgraduée }\end{array}$ & $22(39 \%)$ \\
\hline & $\begin{array}{l}\text { Aucune fonction explicite de responsable } \\
\text { de la formation postgraduée }\end{array}$ & $29(51 \%)$ \\
\hline & Pas de réponse & $6(10 \%)$ \\
\hline
\end{tabular}

Tableau 2

Cours d'introduction aux Mini-CEX dans les établissements.

\begin{tabular}{|c|c|c|}
\hline & $\begin{array}{l}\text { Nombre et taux parmi les } 28 \text { établissements qui ont } \\
\text { les Mini-CEX (plusieurs réponses possibles) }\end{array}$ & ntroduit \\
\hline \multirow[t]{6}{*}{ Soutien de } & Médecin-chef & $17(61 \%)$ \\
\hline & Médecin adjoint & $13(46 \%)$ \\
\hline & Chef de clinique & $14(50 \%)$ \\
\hline & Médecin-assistant & $3(11 \%)$ \\
\hline & Personne & $2(7 \%)$ \\
\hline & $\begin{array}{l}\text { Personne/s d'autre/s institution/s familiarisée/s } \\
\text { avec les Mini-CEX }\end{array}$ & $3(11 \%)$ \\
\hline \multirow[t]{3}{*}{ Forme } & Présentations orales & $24(86 \%)$ \\
\hline & Ateliers interactifs & $12(43 \%)$ \\
\hline & Documents écrits & $13(46 \%)$ \\
\hline \multirow{2}{*}{$\begin{array}{l}\text { Information de méde- } \\
\text { cins-cadres et de } \\
\text { médecins-assistants }\end{array}$} & Commune & $11(39 \%)$ \\
\hline & Séparée & $17(61 \%)$ \\
\hline \multirow[t]{12}{*}{ Matériel utilisé } & Documents de l'IML: & \\
\hline & Powerpoint & $20(71 \%)$ \\
\hline & Film & $12(43 \%)$ \\
\hline & Aide-mémoire & $23(82 \%)$ \\
\hline & Site internet & $19(68 \%)$ \\
\hline & Aucun & $2(7 \%)$ \\
\hline & Documents propres: & \\
\hline & Powerpoint & $10(36 \%)$ \\
\hline & Film & $3(11 \%)$ \\
\hline & Documents écrits & $14(50 \%)$ \\
\hline & Autres (concept de mise en œuvre, description de cas) & $2(7 \%)$ \\
\hline & Aucun & $13(46 \%)$ \\
\hline
\end{tabular}

français et en allemand. Ceux-ci ont été interrogés sur le processus d'introduction dans leur institution environ six mois après avoir suivi le cours. Le taux de réponse a été de $73 \%$, c.à-d. que 57 des 78 médecins ayant participé au cours ont répondu aux questions. Cette enquête a ainsi permis d'approcher 46 des 69 établissements de formation représentés au cours (tabl. 1).

D'après cette enquête, les Mini-CEX ont depuis lors été introduits dans 28 des 46 établissements de formation postgraduée $(=61 \%)$. Dans la plupart des cas, cette procédure a été accompagnée de présentations orales étayées par des diapositives PowerPoint et des informations écrites (tabl. 2). La majorité des teachers ont bénéficié du soutien des médecins-chefs lors de l'introduction, ce qui a manifestement été une condition importante à la bonne mise en œuvre sur place.

Les motifs invoqués par les établissements qui n'ont pas encore introduit les Mini-CEX indiquent clairement qu'ils avaient parfois d'autres priorités (tabl. 3), d'autant plus que ce n'est pas encore obligatoire et que l'introduction des évaluations nationales en matière de qualité au $1^{\text {er }}$ juillet 2012 ou les systèmes d'information des cliniques leur demandent déjà un investissement en temps.

Les motifs mentionnés dans le tableau 3 sont en corrélation avec les réponses concernant les facteurs qui pourraient favoriser l'introduction des Mini-CEX dans les établissements de formation. A la question de savoir ce qui leur manque encore pour l'introduction dans leur établissement, les personnes interrogées des 18 centres de formation ont indiqué qu'elles auraient besoin de plus de temps (11 établissements, 61\%), de plus d'intérêt de la part des médecinscadres et des médecins-assistants à l'introduction des Mini-CEX (6 établissements, 33\%), de plus de soutien de la direction de l'institution (2 établissements, $11 \%$ ) et / ou d'une aide supplémentaire de la part de la SSPP (1 établissement, 5\%) pour l'organisation de l'introduction des Mini-CEX.

La moitié des établissements de formation qui n'ont pas encore introduit les Mini-CEX prévoient des cours d'introduction dans les prochains mois.

\section{Conclusion et perspectives}

La SSPP est la première société de discipline à avoir familiarisé ses médecins-cadres dans toute la Suisse à l'évaluation en milieu de travail (EMiT) en bénéficiant du soutien de l'IML et de l'ISFM. Les cours teach the teacher régionaux censés démultiplier et transmettre les connaissances ont fait leur preuve. Les participants ont apprécié les ateliers interactifs et le dialogue avec les autres formateurs. Les quatre cours proposés en six mois dans trois langues nationales ont permis de sensibiliser $83 \%$ des établissements de formation moyens à grands, ce qui représente un peu plus de la moitié de tous les établissements de formation. A l'été 2012, 61\% de ces institutions travaillaient 


\begin{abstract}
Tableau 3
Motifs invoqués lorsque les Mini-CEX ne sont pas encore introduits.

Nombre et taux parmi les 18 établissements qui n'ont pas encore introduit les Mini-CEX (plusieurs réponses possibles)

La direction de l'institution avait d'autres priorités que l'introduction des Mini-CEX 14 (78\%)

Les Mini-CEX ne sont pas encore obligatoires pour l'obtention du titre de spécialiste 10 (55\%)

Pas le temps d'organiser l'introduction des Mini-CEX

$9(50 \%)$

Aucune demande de la part des médecins-chefs et / ou des médecins-assistants $8(44 \%)$

Autres (par ex. introduction des évaluations en matière de qualité, modifications $3(17 \%)$

structurelles, momentanément pas de médecins en formation postgraduée)

La fonction du teacher a changé dans l'institution

$1(6 \%)$
\end{abstract}

avec les Mini-CEX. Les travaux préalables à l'introduction des Mini-CEX ont pu être réalisés en deux ans environ dès lors que la SSPP avait décidé de les mettre en place. Pour continuer de diffuser l'EMiT et pour maintenir le savoir-faire en la matière dans ses établissements de formation, la SSPP va proposer des cours de mise à jour lors de son congrès annuel. La Commission permanente pour les formations postgraduée et continue (CPF) de la SSPP a commencé une révision de détail du programme de formation postgraduée en psychiatrie et psychothérapie, qui prévoit d'y inclure définitivement les Mini-CEX et dont l'approbation par le comité de la SSPP et l'Assemblée des délégués est prévue pour fin 2012 / début 2013 avec les délais de transition adéquats.

L'oto-rhino-laryngologie a procédé à l'introduction de l'EMiT de manière similaire à la psychiatrie et psychothérapie. D'autres sociétés de discipline ont déjà introduit l'EMiT dans leur programme de formation postgraduée et elles sont sur le point de l'introduire dans leurs établissements de formation. L'IML accompagne les sociétés de discipline lors cette introduction; ensemble, ils adaptent les documents en fonction de la spécialité et planifient les cours teach the teacher. En complément des ateliers interactifs et de la documentation spécifique mise à disposition sur des sites internet, un outil en ligne apportera un soutien audiovisuel utile lors de l'introduction et pour le maintien de l'EMiT. Ces offres bénéficient du soutien financier de l'ISFM mais ce sont les sociétés de discipline qui informent les établissements de formation postgraduée du calendrier et qui fixent les délais de transition. En effet, des conditions-cadres claires facilitent les procédures.

Par ailleurs, la diffusion accrue de cette méthode dans la formation médicale pré- et postgraduée simplifiera également l'introduction et le maintien de l'EMiT dans les différentes sociétés de discipline; celleci deviendra partie intégrante de la formation pré- et postgraduée des médecins. A l'Université de Berne, par exemple, l'EMiT est déjà bien établie. Depuis 2010, les étudiants et les formateurs appliquent déjà régulièrement des évaluations similaires dans la formation pré- et postgraduée. Les possibilités accrues et ciblées d'observer sur place le quotidien hospitalier ainsi que les échanges réciproques sur les objectifs de formation pré- et postgraduée deviendront la routine. L'étude réalisée dans toutes les disciplines auprès des médecins-assistants sur l'évaluation des établissements de formation a montré le besoin d'un feed-back régulier, structuré et donné en temps réel. L'évaluation en milieu de travail est en mesure de combler cette lacune.

\section{Remerciements}

Nous remercions les membres du groupe de travail, Dr G. Berger (psychiatrie intégrée Winterthour-Unterland zurichois), Dr S. Etienne (anciennement Institutions psychiatriques du Valais Romand), Dr D. Georgescu (Services psychiatriques, Argovie), Dr D. Hofer (Services psychiatriques, Thoune), Dr P. Mai (Services psychiatriques, Langenthal) et Dr C. Poppe (clinique psychiatrique privée, Sanatorium Kilchberg) pour la planification du processus d'introduction et la validation des documents relatifs aux Mini-CEX. Nous remercions particulièrement les Docteurs D. Georgescu et C. Poppe, tous deux psychiatres, pour leur participation en tant qu'orateurs aux cours teach the teacher, ainsi que le Dr L. Bolzani, qui s'est aussi chargé des traductions en italien. Et enfin, nous remercions toutes les personnes qui ont répondu à notre enquête. Grâce à elles, nous avons été en mesure de dresser un premier bilan de l'introduction de l'EMiT dans les établissements de formation postgraduée.

\section{Références}

1 Ericsson KA. Deliberate practice and acquisition of expert performance: a general overview. Acad Emerg Med. 2008;15(11):988-94.

2 Van der Horst K, Siegrist M, Orlow P, Berendonk C, Giger M. Démographie, appréciation des études et de la culture de feed-back dans les établissements de formation postgraduée. Résultats de l'enquête de 2008 auprès des médecins-assistants. Bull Méd Suisses. 2010;91(6):203-7

3 Norcini JJ, Blank LL, Arnold GK, Kimball HR. The mini-CEX (clinical evaluation exercise): a preliminary investigation. Annals of internal medicine. 1995;123(10):795-9.

4 Wragg A, Wade W, Fuller G, Cowan G, Mills P. Assessing the performance of specialist registrars. Clinical medicine (London, England). 2003;3(2):131-4.

5 Bielinski D, Eisenhardt K, Frauenfelder B, Fels D, Gitz C, Gysi J et al. Weiterbildung als Attraktivitätsfakor zur Wahl eines medizinischen Fachgebiets - Anspruch und Realität. Bull Méd Suisses. 2011;92(3):53-4.

6 Rogausch A, Berendonk C, Giger M, Bauer W, Beyeler C. Ziel und Nutzen des Arbeitsplatz-basierten Assessments im klinischen Alltag. Eine Einschätzung. Schweiz Med Forum. 2012;12(10):214-7.

7 Berendonk C, Beyeler C, Westkämper R, Giger M Le feed-back structuré dans la formation postgraduée médicale: Mini-CEX et DOPS. Bull Méd Suisses. 2008;89(32):1337-40.

8 Montagne S, Jucker-Kupper P, Berendonk C, Rogausch A, Beyeler C, Giger M. Trois années d'expérience avec l'Evaluation en milieu de travail (Mini-CEX et DOPS) dans la formation postgraduée médicale. Bull Méd Suisses. 2010;91(4):109-11. 